\title{
Identification of Li-Ion Battery Models through Monotonic Echo Serial Networks for coarse data
}

\author{
Antonio Álvarez-Caballero ${ }^{1}$, Cecilio Blanco ${ }^{2}$, Inés Couso ${ }^{3}$ and Luciano \\ Sánchez ${ }^{1}$ \\ ${ }^{1}$ Department of Computer Science, University of Oviedo, Spain. \\ ${ }^{2}$ Department of Electrical Engineering, University of Oviedo, Spain. \\ ${ }^{3}$ Department of Statistics, University of Oviedo, Spain. \\ analca3@gmail.com, cecilio@uniovi.es, couso@uniovi.es, luciano@uniovi.es
}

\begin{abstract}
Monotone transformation models are extended to inaccurate data and are combined with recurrent neural networks in a new battery model that is able to ascertain the health of rechargeable batteries for automotive applications. The presented method exploits the information contained in the vehicle's operational records better than other cutting-edge models, and uses a minimum amount of human expert knowledge. The experimental validation of the technique includes a comparative analysis of batteries in different health conditions, comprising first-principles models and different machine learning procedures.
\end{abstract}

Keywords: Transformation Models; Battery Model; Monotonic Model; Echo State Networks

\section{Introduction}

Battery Management Systems (BMS) are electronic subsystems governing the charge and discharge processes of batteries in Electric Vehicles (EVs). BMSs include hardware and software layers to perform a continuous monitoring of currents, voltages and temperatures of the batteries and implement mathematical models for synthesising the values of different latent variables, such as the State of Charge (SoC) and the State of Health $(\mathrm{SoH})$. These two latent variables are directly related to the range and dependability of the vehicle. Most of EVs already provide an estimation of the SoC of their batteries in the driver's dashboard, but the battery $\mathrm{SoH}$ is not accessible for any of the vehicles that are commercially available in 2017 , because a reliable method for estimating the $\mathrm{SoH}$ from on-vehicle measurements is not available as of yet [1].

In this paper, the $\mathrm{SoH}$ and $\mathrm{SoC}$ of the battery are obtained via computer simulations of a battery model that is kept synchronised with the actual battery state (see Figure 1). The main outcome sought in the presented study is the characteristic Open Circuit Voltage (OCV) of the battery at equilibrium. This is so because the functional dependence between the stored charge and the OCV contains information related to the most common deteriorations of a battery 


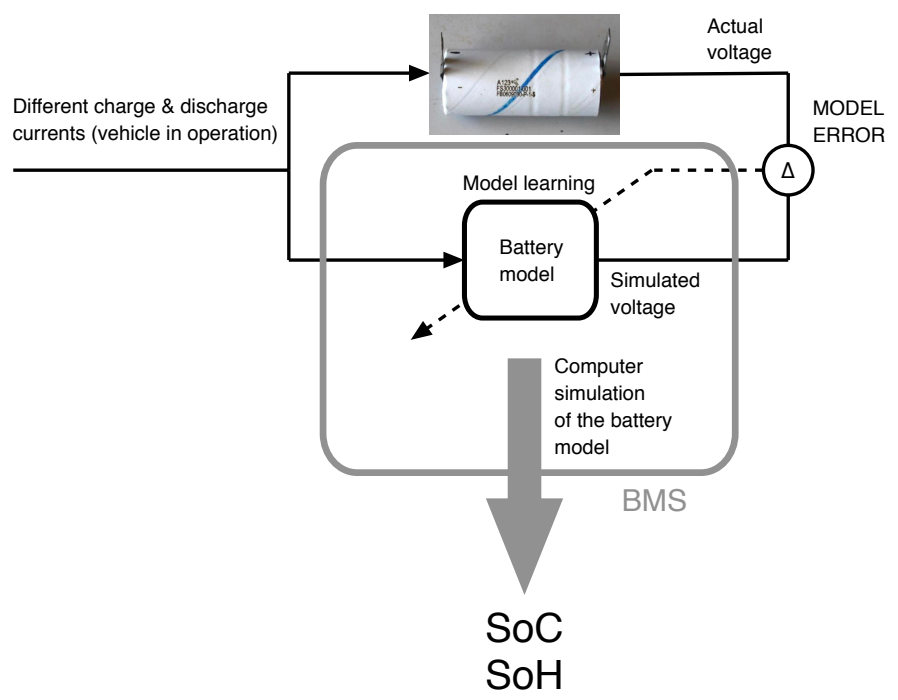

Fig. 1. Battery model synchronised with the actual state of the battery

[2][3], thus a precise estimation of this curve could be easily transformed into the needed health indicator.

Structures similar to that depicted in Figure 1 have been applied in the past to many different battery models. As recently reviewed by $\mathrm{Hu}$ [4], static "equivalent circuit" models (ECMs) are embedded the most in BMSs [5]. Nonstatic or "learning" models are also feasible, including "black boxes" or pure data-driven techniques such as statistical models, time series [6] and neural networks [7]; "grey boxes", such as semi-physical models [8] and "white boxes" or first-principle models, that are based on the knowledge about the electrical and chemical processes that occur while the battery is being operated.

Learning a multi-output dynamical system model is usually intended to minimise the Mean Squared Error (MSE) between the observed output variables and the model. The MSE of a multi-output model is defined as the sum of the MSEs of all output variables. In past works [9] it was proposed that the MSEs of the different predictor variables of the battery model were not combined into a single scalar value but the model was regarded as a multi-criteria problem. In any case, the resulting process had a high computational cost. The learning had to be carried offline in a high performance computer. Hence, the BMS lacked the capability of adapting the model to changes in the battery SoH.

In this paper, a different line of research is followed. ECMs are substituted by Monotone Transformation Models [10], that are a more efficient solution to this problem because of reasons that will be made clear in Section 3. Transformation models relate a pairwise ranking criterion to a monotone transformation function and comprise two components: (a) a dynamical model, whose output is comonotonic with the sought outputs (thus it induces the same ranking in 
the output space as the true system), and (b) a smooth monotone function that maps the output of this dynamical model to the desired output values.

There are some intrinsic advantages in the use of transformation models over single or multi-criteria ECMs. On the one hand, the only assumption made in transformation models is the comonotonicity of an instrumental variable (see Section 2.1 and the second paragraph of Section 3) and the battery voltage. This is a much weaker hypothesis than any of the semi-physical analogies used before, let alone the first-principle models. The simplicity in the assumptions is important because stronger hypothesis (such as the mentioned similarities between an electrical circuit or a flexible vessel and the battery) end up being false when the battery is aged (because the electrochemical processes change, and the battery model comes to be very different). On the other hand, our experiments have shown that the smooth monotone function mentioned before is, with a high accuracy, an affine transformation of the OCV curve. Hence, the computer resources needed for obtaining the health of the battery from the on-vehicle measurements are somewhat reduced. This is very convenient if the model is to be implemented in a BMS.

Notwithstanding, the use of transformation models introduces some difficulties in its own. On-vehicle measurements are imprecise, thus standard statistical rank tests may become inefficient when learning the intermediate dynamical model in this particular application. In the presented method we take advantage of recent rank tests for interval-valued data [11] that alleviate this problem.

The structure of this paper is as follows: in Section 2, transformation models for coarse data are introduced. Section 3 particularises these models to batteries. Section 4 contains experimental tests and discuss the obtained results. The paper concludes in Section 5.

\section{Transformation models for coarse data}

The flexibility of black boxes as universal approximators comes at a cost: the amount of training data needed for complete characterisations of complex nonlinear dynamical systems is hardly attainable. The well known bias-variance dilemma applies here: there must be a balance between the properties of the system that are taken for granted and those that are learned from data. The more knowledge is introduced, the less training instances are needed, but the higher the systematic error may become. Conversely, too flexible models may generalise poorly to unseen areas of the phase space.

Therefore, there is a need for models that make minimal assumptions about the dynamics of the system being studied, in the hope that these assumptions have a wide range of applicability and the systematic error is not increased. In this paper we are interested in those models that encode small amounts of prior knowledge by enforcing the monotonicity of certain nonlinear blocks in the transference function. For instance, any digitally sampled system can be regarded as the composition of a continuous system and a staircase function. Saturations, dead zones, backlashes and different kind of hysteresis also match 
this kind of "monotonic" prior information. The best studied cases are arguably monotonic Hammerstein or Wiener models, that consist in a composition of a linear system with a nonlinear monotonic function [12].

\subsection{Learning monotonic dynamical systems}

Let the pair $(f, \theta)$ comprise a dynamical model defined by a parameter $\theta$, that is applied to an input variable $\left\{u_{t}\right\}_{t}, u_{t} \in \mathbb{R}^{m}$, and a monotonical function $f: \mathbb{R} \rightarrow \mathbb{R}$ that is applied to the output of the dynamical model. The notation " $\left\{u_{t}\right\}_{t}$ " stands for "sequence of values of the variable $u$, indexed by the time $t$ ". The output of the dynamical model is the sequence $\left\{z_{t}(\theta)\right\}_{t}, z_{t}(\theta) \in \mathbb{R}$.

Given a pair of sequences $\left\{y_{t}\right\}_{t}$ (desired output) and $\left\{u_{t}\right\}_{t}$ (observed input), the purpose of the learning algorithm is to find the value of $\theta$ and the function $f$ for which the sequence $\left\{f\left(z_{t}(\theta)\right)\right\}_{t}$ best approximates the desired output of the system $\left\{y_{t}\right\}_{t}$. Prediction error models minimise the MSE between the model output and the desired output,

$$
\operatorname{MSE}(f, \theta)=\sum_{t=d}^{T}\left(f\left(z_{t}(\theta)\right)-y_{t}\right)^{2} .
$$

Transformation models search for the simplest model whose output is comonotonical with the desired output of the system. For example, the MINLIP algorithm for Monotone Wiener Systems [13] solves the following problem:

$$
\begin{aligned}
& \min \operatorname{complexity}(f) \\
& \text { s.t. } f\left(z_{t}(\theta)\right)=y_{t}, \text { for all } t=d+1, \ldots, T
\end{aligned}
$$

where $d$ accounts for the effect of the initial conditions (state of the system at $t=0)$. If noise is to be present, residuals $e_{t}$ are introduced such that $y_{t}=$ $f\left(z_{t}(\theta)+e_{t}\right)$ and the learning algorithm solves the following problem:

$$
\begin{aligned}
& \min \operatorname{complexity}(f) \text { and }\left|e_{t}\right| \\
& \text { s.t. } f\left(z_{t}(\theta)+e_{t}\right)=y_{t}, \text { for all } t=d+1, \ldots, T \text {. }
\end{aligned}
$$

Lastly, $f$ is recovered through an isotonic regression algorithm on the set of pairs $\left\{\left(z_{t}(\theta), y_{t}\right)\right\}_{t}[10]$. The complexity of $f$ is measured through its Lipschitz constant, which is the lowest value $L$ such that

$$
\left|f\left(z_{i}\right)-f\left(z_{j}\right)\right| \leq L\left|z_{i}-z_{j}\right| \text { for all } i, j .
$$

\subsection{Transformation models for coarse data}

Given that $f$ is monotonic, the sequences $\left\{z_{t}(\theta)+e_{t}\right\}_{t=d+1, \ldots, T}$ and $\left\{y_{t}\right\}_{t=d+1, \ldots, T}$ are comonotonic but $\left\{z_{t}(\theta)\right\}$ and $\left\{y_{t}\right\}$ are not comonotonic anymore. However, we expect that the errors $e_{t}$ are small enough thus there still exist two large 
comononotic subsequences of $\left\{z_{t}(\theta)\right\}$ and $\left\{y_{t}\right\}$. Indeed, we can think of a statistical "degree of comonotonicity" defined through a statistical rank test. Since these tests measure the degree to which the ranks of two different variables are the same, the values of the variables do not matter but their order. For instance, the Kendall Tau test [11]

$$
\tau\left(\left\{z_{t}(\theta)\right\}_{t=d+1, \ldots, T},\left\{y_{t}\right\}_{t=d+1, \ldots, T}\right)
$$

consists in sorting the sequence of pairs $\left(z_{t}, y_{t}\right)$ by the first field, and then counting how many of the $y_{t}$ are in order and how many are disordered. These values are substracted and normalised to produce a coefficient that is 1 or -1 for comonotonic or anticomonotonic sequences, and 0 for independent sequences.

An approximation to the best transformation model can be obtained by means of the following multi-criteria optimisation:

$$
\begin{aligned}
& \min \operatorname{complexity}(f) \\
& \min \tau\left(\left\{z_{t}(\theta)\right\}_{t=d+1, \ldots, T},\left\{y_{t}\right\}_{t=d+1, \ldots, T}\right)
\end{aligned}
$$

where the Lipschitz number of $f$ is minimised along with the Kendall Tau distance between the aforementioned sequences. It is remarked that Eqs. (7) and (8) are only an approximation of Eqs. (3) and (4), because the term with $\left|e_{t}\right|$ has been removed.

In this paper, we propose that this term penalising $\left|e_{t}\right|$ is removed from the definition of the "noisy" case, that should depend on a new set of assumptions. Suppose that the desired inputs $u_{t}$ and outputs $y_{t}$ of the model are not accurately perceived but we know that they are enclosed in two sequences of intervals $\bar{u}_{t}$ and $\bar{y}_{t}, u_{t} \in \bar{u}_{t}$ and $y_{t} \in \bar{y}_{t}$ for all $t$. Let the set $\bar{z}_{t}$ be

$$
\bar{z}_{t}\left(\theta,\left\{\bar{u}_{t}\right\}\right)=\left\{z_{t}\left(\theta, u_{t}\right): u_{t} \in \bar{u}_{t}\right\} .
$$

The new set of assumptions for the noisy (set-valued) learning problem is as follows:

$$
\begin{aligned}
& \text { min complexity }(f) \\
& \text { s.t. }\left\{f(z): z \in \bar{z}_{t}\left(\theta,\left\{\bar{u}_{t}\right\}\right)\right\} \subseteq \bar{y}_{t}, \text { for all } t=d+1, \ldots, T .
\end{aligned}
$$

Given that these assumptions do not depend on the unknown values $e_{t}$ anymore, the new problem is equivalent to

$$
\begin{aligned}
& \min \text { complexity }(f) \\
& \min \bar{\tau}\left(\left\{\bar{z}_{t}\left(\theta,\left\{\bar{u}_{t}\right\}\right)\right\}_{t=d+1, \ldots, T} ;\left\{\bar{y}_{t}\right\}_{t=d+1, \ldots, T}\right) .
\end{aligned}
$$

where $\bar{\tau}$ is an set-valued generalisation of the Kendall Tau distance (or any other suitable rank test) [11].

Rank tests for interval-valued data Extending a rank test to set-valued data is problematic because many different ranks may be compatible with sequences of coarse data. For example, consider the following two sequences: 


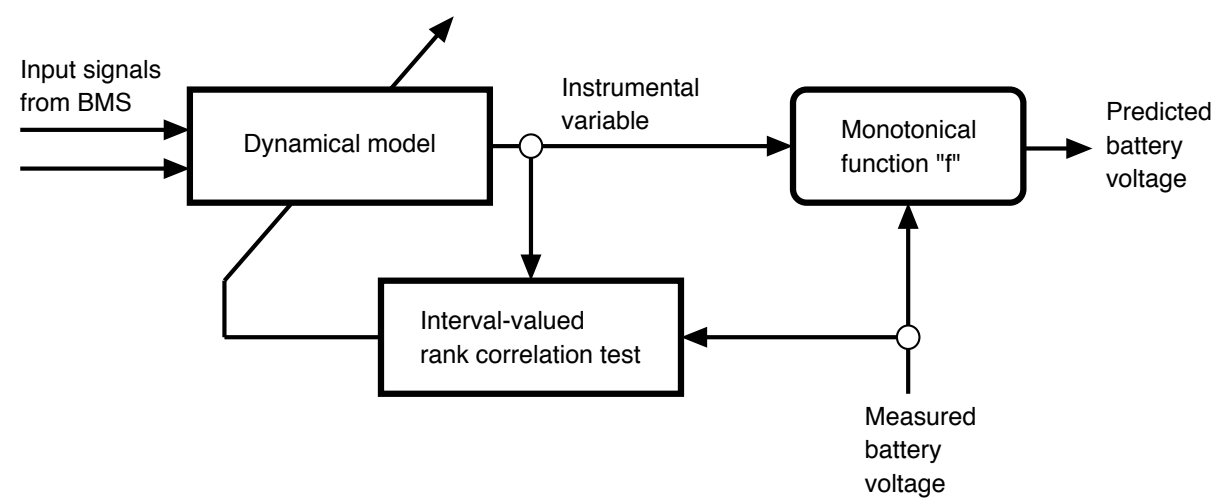

Fig. 2. Transformation model of a battery: the dynamical model depicted in Figure 1 computes an instrumental variable that is comonotonic with the perceived battery voltage. An interval-valued rank test measures the comonotonicity and the dynamical model is adapted if needed. The monotonical function " $\mathrm{f}$ " is obtained with isotonic regression between the instrumental variable and the desired output.

\begin{tabular}{c|cc}
$\mathrm{t}$ & $x_{t}$ & $z_{t}$ \\
\hline 1 & 1 & 2 \\
2 & $1.5 \pm 1$ & 3 \\
3 & 2 & 4
\end{tabular}

Depending on the value of $x_{2}$, these sequences are comonotonic (when $1<x_{2}<$ 2 ), of have discordant pairs (when $x_{2} \leq 1$ or $x_{2} \geq 2$ ). Hence, the value of the rank coefficient is also set-valued. Different generalisations of rank correlation tests to set-valued data exist. For instance, in [11] the Kendall Tau distance for coarse data implements the following extension:

$$
\bar{\tau}\left(\left\{\bar{x}_{t}\right\}_{t=1 \ldots N},\left\{\bar{y}_{t}\right\}_{t=1 \ldots N}\right)=\left\{\tau\left(\left\{x_{t}\right\}_{t=1 \ldots N},\left\{y_{t}\right\}_{t=1 \ldots N}\right): x_{t} \in \bar{x}_{t}, y_{t} \in \bar{y}_{t}\right\} .
$$

Observe that $\bar{\tau}$ is also set-valued thus equation (13) makes reference to the lower bound of this statistic (minimin approach [14]).

Kendall Tau test may not be the most informative when the noise is high and there are many overlapping intervals. A simple alternative consists in replacing the distance $\tau$ in Eq. (14) by a measure of the dispersion of the residual of an isotonic regression of $\{y\}$ with respect to $\{x\}$. This dispersion is also a measure of the "degree of comonotonicity", although it is not easy to ascertain its statistical significance if it was to be used as a statistical test. In the case at hand, however, this is valid because it is only the distance and not the statistical significance that is needed.

\section{Particularisation to battery models}

The particularisation of this structure to battery models is summarised in Figure 2. A nonlinear dynamical model is fed with the operational signals of the vehicle 


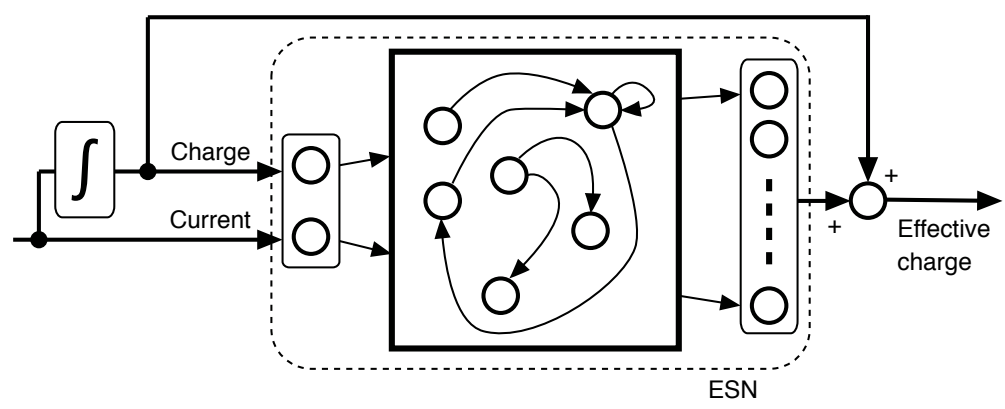

Fig. 3. Dynamical model of the effective charge: the dynamical model of the effective charge comprises a neural network with two inputs: current and charge (integral of the current). The output of the net is the difference between the actual charge and the effective charge.

(current, temperature, etc.) thus its state is kept in sync with the internal state of the true battery. The output of this model is an instrumental variable, with a high degree of comonotonicity with the perceived voltage of the battery. The dynamical model is adapted so as to minimise (the lower bound of) one of the (interval-valued) rank-correlation tests seen in the preceding section. Finally, $f$ is the monotonical function for which the lowest bound of the following set of errors is reached:

$$
\left\{\sum\left\|f\left(z_{t}\right)-y_{t}\right\|^{2} \text { for all } z_{t} \in \bar{z}_{t}(\theta), y_{t} \in \bar{y}_{t}\right\} .
$$

Interestingly enough, the instrumental variable $z$ can be given a physical meaning in this setup. Notice that, if the battery is charged at an infinitesimal pace, the battery voltage is a monotonical function of the battery charge, i.e. the OCV curve. As a consequence of this, if the instrumental variable $z$ is a monotonic transform of the battery charge when the current is small, it results that $f$ must also be a monotone transform of the OCV curve. This property can be attained if the dynamical model is designed as the sum of the actual charge of the battery and an additional term, which is learned from data by means of a learning algorithm that promotes that the norm of the output of the model is as low as possible.

If the learned model (with $f$ being a transform of the OCV curve) is subjected to high charge or discharge currents, $z$ can be understood as an "effective charge": the charge that an hypothetical battery at equilibrium should store for having the same voltage as the battery being modelled. Hence the dynamical model would be predicting the diffusion and over-potential effects [15] in the battery.

Since a human-understandable definition of these diffusion and over-potential effects is not needed for assessing the health of the battery, a black-box (a recurrent neural network) has been used in this paper: an Echo State Network (ESN) with a linear activation layer [16]. Recurrent networks, and particularly 
ESNs, can learn models where the output depends on the input and also on the past history of the system, as happens with batteries. The disposition of the elements in the presented model is displayed in Figure 3. It is remarked again that the neural network is not modelling the effective charge but the difference between the effective charge and the actual charge (thus the output of the net tends to zero if the current is sustainedly low). Observe also that the weights of the output layer cannot be determined by ridge regression [17] (because the desired output is unknown). A gradient descent algorithm is used for minimising a rank correlation test between (a) the sum of the charge and the output of the net and (b) the voltage of the battery, with an additional term penalising the absolute value of the integral in time of the net output.

Lastly, let us explain the meaning assigned to the intervals $\bar{y}_{t}$ and $\bar{u}_{t}$ in this context. On the one hand, the intervals $\bar{y}_{t}$ reflect the tolerance of the sensors in the vehicle. If $y_{t}$ is the output value that is actually observed at the battery, then $\bar{y}_{t}=y_{t} \pm \epsilon$ for a certain $\epsilon$ that depends on the accuracy of the sensor. On the other hand, the spread of the intervals $\bar{u}_{t}$ handles the tolerance of the sensors and also the effects of the unknown inputs to the model and the possible lack of flexibility of the parametric family of the models; $\widetilde{z}_{t}\left(\theta,\left\{\bar{u}_{t}\right\}\right)$ must be wide enough to compensate for the systematic error of the model. The spreads of $\bar{y}_{t}$ and $\bar{u}_{t}$ are, therefore, design parameters that depend on the tolerance of the particular sensors being used in the BMS and the properties of the parametric family of the dynamical model.

\section{Empirical study and discussion}

The experimentation in this section is designed to find out whether the proposed data-driven method is competitive with state-of-the-art battery models. The experiments were conducted on three different batteries: Battery \#1 is a $42 \mathrm{Ah}$ pouch battery from European Batteries, and Batteries \#2 and \#3 are cylindrical commercial LFP cells manufactured by A123 Systems, with 2.3 Ah name plate capacity. The following conditions are analysed:

1. Influence of the current. Battery \#1 was used for this purpose, and was charged at 42, 21, 14, 8.4 and 1.68 Amps (these currents are named C1, C2, C3, C5 and C25). It is expected that the model accuracy is best for C25 and degrades for the higher currents.

2. Influence of the ageing of the battery. Batteries \#2 and \#3 were subjected to 6000 charge/discharge cycles and different experiments were programmed at the beginning of their lives, at half life (3000 cycles) and at the end of their useful life (6000 cycles). Battery \#3 had an abnormal deterioration (electrodeposition). Battery \#2 had a normal ageing with a gradual reduction of the capacity until the end of its life.

3. Influence of the technology. Battery \#1 is a pouch battery; \#2 and \#3 are cylindrical, and the capacities are also different; Battery \#1 is a large cell (42Ah, used for instance in battery-electric buses) and the other cells are 
much smaller (2.3Ah, used for instance in BMW ActiveHybrid 3 HEVs or the Chevrolet Spark EV).

The $\mathrm{LiFePO}_{4}$ (LFP) pouch battery from European Batteries has a rated capacity of $42 \mathrm{Ah}$ when discharged at $8.4 \mathrm{Amps}$. The average operating voltage is $3.2 \mathrm{~V}$. The discharge and charge cut-off voltages are $2.5 \mathrm{~V}$ and $3.65 \mathrm{~V}$ respectively. The dimensions in $\mathrm{mm}$. are $275 \times 166.5 \times 13.3$. The cell weights $1010 \mathrm{~g}$. The cylindrical battery from A123 Systems has a rated capacity of 2.3 Ah when discharged at 2.3 Amps. The average operating voltage is $3.3 \mathrm{~V}$. The discharge and charge cut-off voltages are $2 \mathrm{~V}$ and $3.6 \mathrm{~V}$ respectively. The dimensions in $\mathrm{mm}$ are $\phi 26 \times 65$. The cell weights $76 \mathrm{~g}$.

Tests are conducted in a SBT 10050 battery test system from PEC and an ICP 750 climate chamber from Memmert. The ambient temperature was $23^{\circ} \mathrm{C}$. The OCVs of the three batteries have been measured through the "voltage relaxation" method [18]. This method consists in charging the battery in small steps (10\% of the capacity) and then applying a constant voltage until the current is smaller than a threshold to ensure a full charge (or discharge). Each of these steps is followed by a rest period of some hours, after which the OCV voltage is measured. It is remarked that train and test datasets are not obtained with cross validation. The learning algorithm uses on-vehicle data (charge and discharge at different currents). A neural network is trained to maximise the rank correlation between (a) the sum of its output and the actual charge of the battery and (b) the measured voltage of the battery at the vehicle. Once the neural network is trained, the function $f$ is obtained by isotonic regression between the sequences (a) and (b). This function $f$ is subsequently compared to the actual OCV curve of the battery, that has been independently obtained at the laboratory by means of a relaxation experiment, as mentioned before. The ECMs tabulated in the forthcoming tables are the mean squared differences between the values of $f$ and the true OCV curve.

Three different setups of the proposed model were used:

1. ESN- $\tau$ : Combination of an ESN (reservoir of size 1000, linear output layter) with the extension of Kendall Tau to interval valued data, as defined in Ref. [11].

2. ESN- $x$ : Combination of the same ESN with the isotonic regression-based measure of comonotonicity. This is the variance of the residual of an isotonic regression with zero tolerance for the output variable and $10 \%$ tolerance for the input variables.

3. ESN-xy: The same as before, but the tolerance is $10 \%$ for the inputs and $1 \%$ for the outputs.

Apart from the three proposed model setups, the following eight models are included in the study:

1. Abu-Sharkh's method [19]. State-of-the-art method for determining the OCV of a battery from operational data, based on a first-principles model of the battery. 
Table 1. Influence of the charging current. Average quadratic error of OCV, obtained from RNN, LSTM, ESN, $\gamma$-ESN, ANFIS, and ARIMAX

\begin{tabular}{cccccc}
\hline & C25 & C5 & C3 & C2 & C1 \\
\hline Abu-Sharkh & $\mathbf{0 . 0 0 0 3}$ & 0.0094 & 0.0080 & 0.0084 & 0.0110 \\
Xu & 0.0006 & 0.0086 & 0.0146 & 0.0153 & 0.0073 \\
\hline LSTM & 0.0077 & 0.0301 & 0.0070 & 0.0066 & 0.0064 \\
LSTM-dropout & 0.0100 & 0.0295 & 0.0067 & 0.0083 & 0.0093 \\
ESN & 0.0056 & 0.0326 & 0.0083 & 0.0106 & 0.0083 \\
$\gamma$-ESN & 0.1553 & 0.0854 & 0.0279 & 0.0132 & 0.0212 \\
ANFIS & 0.2026 & 0.2016 & 0.0731 & 0.0595 & 0.0334 \\
ARIMAX $(2,1)$ & 0.0127 & 0.0391 & 1.0153 & 0.0165 & 0.0202 \\
\hline ESN- $\tau$ & $\mathbf{0 . 0 0 0 3}$ & $\mathbf{0 . 0 0 0 2}$ & $\mathbf{0 . 0 0 0 4}$ & $\mathbf{0 . 0 0 0 4}$ & $\mathbf{0 . 0 0 1 3}$ \\
ESN- $x$ & $\mathbf{0 . 0 0 0 3}$ & 0.0003 & 0.0007 & 0.0007 & 0.0018 \\
ESN- $x y$ & $\mathbf{0 . 0 0 0 3}$ & 0.0003 & 0.0007 & 0.0007 & 0.0018 \\
\hline
\end{tabular}

2. Xu's method [20]. Another specialised method that is based on Randles' equivalent circuit.

3. LSTM [21]. A LSTM recurrent neural network with 20 hidden nodes with recurrent connections followed by a linear layer. The net is primed with two sequences of inputs: the current and the charge.

4. LSTM-dropout [22]. A regularised LSTM network where randomly chosen network units are masked during training.

5. ESN [23]. Echo State Network, with a reservoir of size 1000, and a linear feed-forward layer trained with ridge regression.

6. $\varphi$-ESN [24] An ESN with an additional nonlinear feed-forward layer, where the non-recurrent layers are trained with the Adam algorithm [25].

7. ANFIS (Adaptive-Network-Based Fuzzy Inference System)[26] in a NARX configuration.

8. ARIMAX $(2,1)$ : Auto Regressive Integrated Moving Average with Explanatory Variable time series, with orders $\mathrm{AR}=2, \mathrm{MA}=1$.

The results of the study are displayed in Tables 1,2 and 3. The influence of the charge/discharge rate in the accuracy of the sensor is studied in Table 1. Observe that the accuracy of all methods degrades for high currents. Remarkably, the presented method was able to improve over battery specific algorithms, and keep the $\mathrm{C} 25$ accuracy for discharges at high currents. In addition to this, the performance of the new approach is better by an order of magnitude than any of the other methods for currents C3 and C2. However, none of the methods achieved satisfactory results for $\mathrm{C} 1$.

Table 2 measures the accuracy of the model when the batteries are at the beginning, middle and end of their lives and the ageing process has been uniform (without abnormal deterioration). In all cases the charging current is C25. In these experiments, Abu-Sharkh and Xu's methods were only evaluated in their 
Table 2. Influence of the number of cycles without electrodeposition. Average quadratic error of OCV, obtained from RNN, LSTM, ESN, $\gamma$-ESN, ANFIS, and ARIMAX

\begin{tabular}{cccc}
\hline & New battery & Middle life & End of Life \\
\hline Abu-Sharkh & 0.0003 & 0.0008 & 0.0009 \\
Xu & 0.0008 & 0.0016 & 0.0015 \\
\hline LSTM & 0.0016 & 0.0027 & 0.0069 \\
LSTM-dropout & 0.0015 & 0.0035 & 0.0033 \\
ESN & 0.0060 & 0.0131 & 0.0125 \\
$\gamma$-ESN & 0.0132 & 0.0120 & 0.1972 \\
ANFIS & 0.0573 & 0.0913 & 0.0808 \\
ARIMAX $(2,1)$ & 0.0494 & 0.0619 & 0.0603 \\
\hline ESN- $\tau$ & 0.0010 & 0.0012 & 0.0014 \\
ESN- $x$ & $\mathbf{0 . 0 0 0 2}$ & 0.0006 & $\mathbf{0 . 0 0 0 3}$ \\
ESN- $x y$ & 0.0003 & $\mathbf{0 . 0 0 0 5}$ & 0.0004 \\
\hline
\end{tabular}

Table 3. Influence of the number of cycles with electrodeposition. Average quadratic error of OCV, obtained from RNN, LSTM, ESN, $\gamma$-ESN, ANFIS, and ARIMAX

\begin{tabular}{cccc}
\hline & New battery & Middle life & End of Life \\
\hline Abu-Sharkh & $\mathbf{0 . 0 0 0 2}$ & 0.0008 & 0.0010 \\
Xu & 0.0007 & 0.0016 & 0.0018 \\
\hline LSTM & 0.0026 & 0.0026 & 0.0031 \\
LSTM-dropout & 0.0022 & 0.0019 & 0.0029 \\
ESN & 0.0054 & 0.0212 & 0.0639 \\
$\gamma$-ESN & 0.0062 & 0.1506 & 0.0343 \\
ANFIS & 0.0639 & 0.1709 & 0.1549 \\
ARIMAX $(2,1)$ & 0.0620 & 0.0447 & 0.0828 \\
\hline ESN- $\tau$ & $\mathbf{0 . 0 0 0 2}$ & 0.0011 & 0.0004 \\
ESN- $x$ & 0.0003 & 0.0004 & 0.0005 \\
ESN- $x y$ & $\mathbf{0 . 0 0 0 2}$ & $\mathbf{0 . 0 0 0 3}$ & $\mathbf{0 . 0 0 0 2}$ \\
\hline
\end{tabular}


most favourable configuration, i.e. for charges lower than $80 \%$ of the capacity of the battery. The accuracy of the new method is always comparable or better than that of the specific methods, showing also that the presented method is not negatively influenced by the battery age.

Lastly, Table 3 measures the accuracy of the model at the beginning, middle and end of life when an abnormal deterioration takes place (an electrodeposition happened at some point between the middle and the end of the life). There are not appreciable differences between the accuracy of the soft sensor in this case and the results in the preceding Table 2 .

\section{Concluding remarks}

A monotonic transformation model is used for obtaining the OCV curve of a battery while it is not at equilibrium. This model comprises a recurrent neural network and a monotonic function that is obtained via isotonic regression. The neural network is trained by minimising the lower bound of a statistical rank test for coarse data, according to a novel interval-valued characterisation of the tolerances of the sensors and the systematic errors inherent to the parametric definition of the dynamical model. Apart from the improvement in the accuracy of the model shown in the results, the new characterisation simplifies the optimisation problem by removing the noise-related terms. The size reduction allows to implement the method in devices with reduced computational power.

The results were validated with two different types of LFP batteries for automotive applications, for different states of health and charge rates. Two firstprinciple models, five recurrent neural networks and statistical time series were compared to the presented method. It was concluded that transformation models for coarse data improve existing methods by a large margin when the charge current is high, without a significant influence of the state of health of the battery.

In future works it is planned that the Monte-Carlo implementation of the regression-based rank test is improved by means of an interval-valued implementation of the PAVA algorithm for isotonic regression. Although the computational demand of the current algorithm is lower than that of the previous intelligent models, the power of current BMS still may be insufficient for performing the learning online.

\section{Acknowledgements}

This work has been funded by the Spanish Ministry of Science and Innovation (MICINN) and the Regional Ministry of the Principality of Asturias under Grants TIN2014-56967-R, TIN2017-84804-R, DPI2013-046541-R and FC15-GRUPIN14-073. 


\section{References}

1. Waag, W., Fleischer, C., Sauer, D.U.: Critical review of the methods for monitoring of lithium-ion batteries in electric and hybrid vehicles. Journal of Power Sources 258 (July 2014) 321-339

2. Birkl, C.R., Roberts, M.R., McTurk, E., Bruce, P.G., Howey, D.A.: Degradation diagnostics for lithium ion cells. Journal of Power Sources 341 (2017) 373-386

3. Weng, C., Sun, J., Peng, H.: A unified open-circuit-voltage model of lithium-ion batteries for state-of-charge estimation and state-of-health monitoring. Journal of Power Sources 258 (2014) 228 - 237

4. Hu, X., Li, S., Peng, H.: A comparative study of equivalent circuit models for Li-ion batteries. Journal of Power Sources 198 (jan 2012) 359-367

5. Zhang, C., Li, K., Pei, L., Zhu, C.: An integrated approach for real-time modelbased state-of-charge estimation of lithium-ion batteries. Journal of Power Sources 283 (jun 2015) 24-36

6. Saha, B., Goebel, K., Christophersen, J.: Comparison of prognostic algorithms for estimating remaining useful life of batteries. Transactions of the Institute of Measurement and Control 31(3-4) (2009) 293-308

7. Lu, L., Han, X., Li, J., Hua, J., Ouyang, M.: A review on the key issues for lithiumion battery management in electric vehicles. Journal of Power Sources 226 (2013) $272-288$

8. Sánchez, L., Couso, I., González, M.: A design methodology for semi-physical fuzzy models applied to the dynamic characterization of LiFePO4 batteries. Applied Soft Computing 14 (January 2014) 269-288

9. Echevarría, Y., Sánchez, L., Blanco, C.: Assessment of multi-objective optimization algorithms for parametric identification of a li-ion battery model. In: International Conference on Hybrid Artificial Intelligence Systems, Springer (2016) 250-260

10. Belle, V.V., Pelckmans, K., Suykens, J.A.K., Huffel, S.V.: Learning Transformation Models for Ranking and Survival Analysis. Journal of Machine Learning Research 12(Mar) (2011) 819-862

11. Couso, I., Strauss, O., Saulnier, H.: Kendall's rank correlation on quantized data: An interval-valued approach. Fuzzy Sets and Systems (2017)

12. Schetzen, M.: Nonlinear System Modelling and Analysis from the Volterra and Wiener Perspective. In: Block-oriented Nonlinear System Identification. Springer London, London (2010) 13-24

13. Pelckmans, K.: MINLIP for the identification of monotone Wiener systems. Automatica 47(10) (2011) 2298-2305

14. Couso, I., Sánchez, L.: Machine learning models, epistemic set-valued data and generalized loss functions: An encompassing approach. Information Sciences $\mathbf{3 5 8}$ (2016) 129-150

15. Gallagher, K.G., Dees, D.W., Jansen, A.N., Abraham, D.P., Kang, S.H.: A volume averaged approach to the numerical modeling of phase-transition intercalation electrodes presented for lixc6. Journal of The Electrochemical Society 159(12) (2012) A2029-A2037

16. Verstraeten, D., Schrauwen, B., Haene, M., Stroobandt, D.: An experimental unification of reservoir computing methods. Neural networks 20(3) (2007) 391-403

17. Lukoševičius, M.: A practical guide to applying echo state networks. In: Neural networks: Tricks of the trade. Springer (2012) 659-686

18. Pei, L., Lu, R., Zhu, C.: Relaxation model of the open-circuit voltage for state-ofcharge estimation in lithium-ion batteries. IET Electrical Systems in Transportation 3(4) (2013) 112-117 
19. Abu-Sharkh, S., Doerffel, D.: Rapid test and non-linear model characterisation of solid-state lithium-ion batteries. Journal of Power Sources 130(1-2) (2004) 266-274

20. Xu, J., Cao, B., Chen, Z., Zou, Z.: An online state of charge estimation method with reduced prior battery testing information. International Journal of Electrical Power \& Energy Systems 63 (2014) 178-184

21. Hochreiter, S.: The vanishing gradient problem during learning recurrent neural nets and problem solutions. International Journal of Uncertainty, Fuzziness and Knowledge-Based Systems 6(02) (1998) 107-116

22. Gal, Y., Ghahramani, Z.: A theoretically grounded application of dropout in recurrent neural networks. In: Advances in Neural Information Processing Systems. (2016) 1019-1027

23. Maass, W., Natschläger, T., Markram, H.: Real-Time Computing Without Stable States: A New Framework for Neural Computation Based on Perturbations. Neural Computation 14(11) (2002) 2531-2560

24. Gallicchio, C., Micheli, A.: Architectural and markovian factors of echo state networks. Neural Networks 24(5) (2011) 440-456

25. Kingma, D.P., Ba, J.: Adam - A Method for Stochastic Optimization. In: The International Conference on Learning Representations (ICLR). (2014)

26. Jang, J.S.: Anfis: adaptive-network-based fuzzy inference system. IEEE Transactions on Systems, Man, and Cybernetics 23(3) (1993) 665-685 